\title{
Nanomedicine for Cancer Therapy
}

Mansoor M. Amiji

Received: 18 August 2010 / Accepted: 25 August 2010 / Published online: 16 September 2010

(C) Springer Science+Business Media, LLC 2010

Nanotechnology has tremendous potential in disease prevention, diagnosis, imaging, and therapy. The United States National Institutes of Health (NIH) has defined nanomedicine as a molecular-scale intervention for prevention, diagnosis, and treatment of diseases. The National Cancer Institute's (NCI) Alliance for Nanotechnology in Cancer (http://nano.cancer.gov) was initiated in 2004 to: 1) provide researchers with the opportunity to study and manipulate macromolecules in real time and during the earliest stages of cancer progression, 2) enable rapid and sensitive detection of cancer-related molecules, enabling scientists to detect molecular changes even when they occur only in a small percentage of cells, and 3) support entirely novel and highly effective therapeutic agents.

In this Pharmaceutical Research theme issue on Nanomedicine for Cancer Therapy, world-renowned scientists, engineers, and clinical researchers have provided a glimpse into the possibilities of nanotechnology applications for cancer. Dr. Piotr Grodzinski and colleagues from the Alliance for Nanotechnology in Cancer at NCI provide a unique perspective from the Alliance's efforts in fostering nanotechnology applications in cancer therapeutics, with special emphasis on delivery challenges and toxicities associated with cytotoxic chemotherapies. Professor Mark Davis and colleagues from the California Institute of Technology further the discussion on challenges and opportunities of clinical translation. They focus on technologies that have been investigated and discuss numerous

M. M. Amiji $(\square)$

Northeastern University

Boston, MA, USA

e-mail: m.amiji@neu.edu types of therapeutic molecules and passive versus active targeting strategies employed. Professor Leaf Huang and his colleagues from the University of North Carolina at Chapel Hill provide a comprehensive review on cancer and the immune system. Nanotechnology can provide elegant solutions to enhance cancer vaccine efficacy and approaches for immune-modulation. Cancer vaccines and immunotherapy are also addressed in the article by Professor Aliasgar Salem and colleagues from the University of Iowa. They discuss strategies to target nanoparticulate carriers to antigen-presenting cells in the body. Professor Kattesh Katti and colleagues from the University of Missouri in Columbia present their work on the development of multifunctional gold nanoparticles synthesized using bio-friendly reagents for tumor-targeted imaging and early detection. The use of inorganic metal-based nanotechnology for imaging and therapy is further discussed in a review by Dr. Priyabrata Mukherjee and colleagues from the Mayo Clinic. In addition to using gold-, silver-, and platinum-based nanoparticles as vehicles for targeted imaging and drug delivery, these investigators have also found that the metals inherently have various therapeutic functions that could benefit cancer treatment. Dr. Zheng-Feng Duan and colleagues from Mass General Hospital and Northeastern University review the application of nanotechnology for cancer therapy with a specific focus on osteosarcomas, which are relatively aggressive tumors with a very high probability of resistance. Professor Mauro Ferrari and his colleagues from the University of Texas Health Science Center discuss the use of nanochannel fabricated silicon membrane devices for controlled release for metronomic therapy. Finally, Professor Vladimir Torchilin and colleagues from Northeastern University discuss their study on the evaluation of palmitoyl 
ascorbate-encapsulated micelles for tumor-targeted delivery and efficacy in $4 \mathrm{~T} 1$ mammary tumor model.

The success of any theme issue of the journal depends heavily on the contributions of the various manuscripts. I am deeply grateful to all of the individual contributing authors who have supported this project. Each manuscript was reviewed by at least three independent reviewers, and their comments were essential in improving the quality of manuscripts for the theme issue on Nanomedicine for Cancer Therapy. Finally, special thanks are due to all the wonderful individuals from the Pharmaceutical Research Editorial Office, including Editorial Assistant Ms. Rachel Lucke, who efficiently managed the project; Special Features Editor, Professor Ram Mahato from the University of Tennessee; and the Editor-in-Chief Professor Peter Swaan from the University of Maryland.

Any suggestions or points of discussion can be directed to the Theme Issue Editor, Professor Mansoor Amiji, by email atm.amiji@neu.edu.

\section{INTERVIEW WITH DR. MANSOOR M. AMIJI}

\section{What do you think holds the key to your success as a pharmaceutical scientist?}

As an academic scientist and educator for the past 17 years, I believe that a strong educational background, based on fundamental understanding of basic scientific principles, working with high-caliber postdocs and students, a sharp focus on problem solving combined with an inquisitive attitude, and a willingness to engage and collaborate with others from different scientific fields are some of the keys to my success.

I graduated from Northeastern University in Boston, MA with a BS in pharmacy at a time when basic and applied pharmaceutical sciences education was highly valued. Today, I feel that pharmacy education in the United States has moved far more into the clinical side, especially at the expense of laboratory-based pharmaceutical science courses. These courses are essential in training future pharmaceutical science researchers and educators. For my PhD studies, I went to Purdue University in West Lafayette, IN, and again I received excellent education and training with strong emphasis on the fundamentals. My PhD dissertation at Purdue focused on blood compatibility evaluation of surface-modified biomaterials. I am immensely proud of the 80 or so postdocs and students that I have trained over my career at Northeastern. Each one of them is very successful in their own careers and has made significant contributions in pharmaceutical sciences.
I have been able to focus on the problems that we are pursuing to solve and try to come up with imaginative, yet practical, solutions. In some of the solutions, it is important to also take a contrarian view from whatever the consensus in the field may be. I also believe strongly that the solutions to some of our most daunting problems will come from individuals who are at the interface of various disciplines. It is critical to have a deeper understanding of other scientific and technological fields and interact with other scientists, engineers, and clinicians on a regular basis. Embracing inter- and multi-disciplinary approaches is essential to the success of any academic or industrial scientist. For instance, I make a point to include a clinician or a clinical researcher in my doctoral students' thesis committees. Having a perspective from the clinical side adds tremendous value to the student's education and training by making sure that the solutions that they pursue have a realistic expectation of being translatable and potentially able to address the challenges in the clinic.

\section{What do you consider to be your key research accomplishments?}

Oral Anti-infective Therapy: After coming to Northeastern in 1993, I started to work on development of an oral drug delivery platform for stomach-specific anti-infective therapy in treating Helicobacter pylori infection. We identified a number of factors that are important to ensure success of local therapy and went on to develop chitosan-based hydrogels that had $\mathrm{pH}$-sensitive swelling and drug release properties. Antibiotic and non-antibiotic anti-H. pylori agents have been encapsulated in the chitosan-based hydrogels, and the systems have been evaluated in both gerbil and rat models. Initial funding for these studies came from NIH through an SBIR Phase 1 grant with Eos Pharmaceuticals. A number of postdocs and graduate students worked on the projects. Additional funding was also received from Medix Corporation in Mexico City, Mexico. This research has led to several very successful collaborations including with Dr. David Cave at Saint Elizabeth Medical Center in Boston, MA, Dr. Masae Tatematsu's group at the Aichi Cancer Center Research Institute, Nagoya, Japan, and more recently, Dr. Saul Tzipori at the Tufts Veterinary School in Grafton, MA.

Additional effort in anti-infective therapy is currently focused on development of orally delivered mucosal vaccination strategies using multiple emulsion formulations. We have selected peptide- and plasmid DNA-based vaccination approaches for oral delivery in the Peyer's patch region of the small intestine. Proof-of-concept for this approach was initiated with ovalbumin encapsulated in squalane-containing multiple emulsion formulations. These 
studies are ongoing through collaborations with Novartis Vaccine and Diagnostics group in Cambridge, MA.

Nano-emulsions Delivery Systems: Oil-in-water emulsions are a highly versatile platform for delivery of hydrophobic agents. Using edible oils that are rich in omega- 3 and omega- 6 polyunsaturated fatty acids, we have observed that nano-sized oil droplets in these emulsions can facilitate drug delivery in hard-to-reach areas of the body upon both oral and systemic administration. We initially developed the formulations using an ultrasound method but have refined the manufacturing and scale-up using a highpressure microfluidic system obtained through collaborations with Microfluidics Corporation in Newton, MA. We have observed that omega-3 fatty acid-containing oils enhance brain delivery of therapeutics. Surface engineering of the nano-emulsions can provide additional functionality through attachment of targeting ligand or imaging agents. The nano-emulsion technology has been licensed out of Northeastern to Nemucore Medical Innovations, Inc. in Wellesley, MA.

The NGI Alliance for Nanotechnology in Cancer established the Nanotechnology Characterization Laboratory (NCL) in Frederick, $\mathrm{MD}$ for in vitro and in vivo preclinical evaluations of nanosystems. We have established fruitful collaborations with NCL on development of nanoemulsions for brain tumor imaging and therapy. Additional funding for nano-emulsion-based delivery systems for CNS therapy was recently obtained through an R21 grant from the National Institute of Neurological Diseases and Stroke.

Non-condensing Nucleic Acid Delivery Systems: After getting tenure and being promoted to Associate Professor in 1999, I took a sabbatical leave and had the distinct privilege of working in Professor Bob Langer's lab at MIT for a short period of time on synthesis and evaluation of poly(betaamino ester)s for non-viral gene delivery. With Dr. David M. Lynn, a postdoc in Bob's lab (now on the faculty at University of Wisconsin, Madison), we evaluated several different kinds of poly(beta-amino ester)s made by reacting primary and secondary amines with diol-diacrylate. I realized that almost all of the non-viral approaches for gene therapy were focused on the use of cationic lipids and polymers. The majority of these systems were not very efficient in DNA delivery upon systemic administration and had high degree of toxicity concerns.

To overcome the limitations of cationic lipids and polymers, we hypothesized that neutral or even negatively charged hydrophilic biodegradable polymers would be better vehicles for systemic nucleic acid delivery. Since 2001, we have been working on different types of nanoparticulate formulations of non-condensing gene delivery systems. Using type B gelatin, we formulated nanoparticles encapsulating reporter protein-expressing plasmid DNA and then went on to study soluble VEGF-R1 (sFlt-1) transgene expression in an orthotopic breast cancer model (NCI R01 grant support). Additionally, gelatin nanoparticles encapsulated further in polymeric microsphere systems were evaluated for oral gene delivery and transfection using murine IL-10 expressing plasmid DNA in an inflammatory bowel disease model (funded by National Institute of Diabetes and Digestive and Kidney Diseases R01 grant). More recently, we are exploring the use of alginate-based nanoparticles made specifically to target macrophages upon systemic administration for antiinflammatory gene therapy. This work has also led to several research collaborations in the Boston area.

In addition to plasmid DNA, we have also examined siRNA delivery using non-condensing polymeric systems. With Dr. Zheng-Feng Duan and his colleagues at Mass General Hospital in Boston, we are investigating the use of dextran-based self-assembled nanosystems for gene silencing and therapy of multidrug resistant tumors. Postdocs and graduate students in the lab are also working on other areas of siRNA delivery, including TNF-a gene silencing in inflammatory bowel disease and HIF-la gene silencing in cancer.

Nano-therapeutic Strategy to Overcome Tumor Resistance: In 2005, I received the Cancer Nanotechnology Platform Partnership (CNPP) grant from the Alliance for Nanotechnology for Cancer to evaluate multifunctional nanoparticle delivery system to overcome tumor resistance. Previously, we had developed biodegradable polymeric systems for tumor-targeted delivery of hydrophobic drugs, such as paclitaxel and tamoxifen. With the CNPP grant from 2005 to 2010 as well as an ARRA Administrative Supplement funding, we have been able to show that combination therapeutic strategy that enhances drug delivery efficiency and can also affect the cellular phenotype in resistance tumors will have a more profound impact on the clinical management of refractory cancers. With ceramide co-therapy, we have shown that nanoparticulate formulations can enhance apoptotic cell-kill efficiency in multidrug resistant (MDR) breast and ovarian cancer models. This work has led to important collaborations with Dr. Michael Seiden when he was at Mass General Hospital (now CEO of Fox Chase Cancer Center in Philadelphia, PA) and Dr. Zhen-Feng Duan. More recently, we have expanded our effort to evaluate mitochondria-targeted ceramide derivatives as more effective agents for lowering tumor apoptotic threshold (funded by NCI R21 grant).

Further studies are ongoing in the lab to evaluate the role of hypoxia in the development of tumor resistance. We hypothesized that lack of oxygen and nutrients as well as low $\mathrm{pH}$ in regions of hypoxic solid tumors lead to a more resistant cellular phenotype based on adaptations to the 
harsh microenvironment. Aerobic tumor metabolism and the associated Warburg's effect and the role of interstitial matrix are other areas of interest in understanding the development of refractory disease.

\section{What was the turning point in your career?}

There have been several important transformative points in my career. First was the choice of coming to academia in 1993, and especially Northeastern University in Boston, after working for a short period of time at Columbia Research Labs in Madison. Although the primary reason at the time was job security, the choice of an academic career has suited me very well. I love interacting and engaging with students, be it in a large classroom for lectures or in a one-to-one setting of the research lab.

The second turning point was when I had the amazing opportunity to work in Bob Langer's lab at MIT in 2000. The proximity of Bob's lab and his continuous generosity have been transformative to our research efforts and productivity. I have been able to forge great collaborations with academic investigators and find industrial partnerships due to the experiences from MIT. I particularly want to note our long-standing collaborations with Dr. Steve Little, who completed his $\mathrm{PhD}$ under Bob and is now on the faculty at University of Pittsburgh.

The third issue in supporting my career development was being able to get sustained financial support from NIH and other funding sources. Financial support through the R01 and other grants that I have received over the last decade total approximately $\$ 10$ million. This funding has allowed me to hire and retain excellent postdocs and students and to purchase equipment, chemicals, and supplies to carry out our projects. I have seen too many ideas and careers of academic investigators negatively affected due to lack of funding.

The fourth important point is the opportunity that I have had to take more administrative and leadership roles at the department, School of Pharmacy, and university. I was the Academic Coordinator of the university-wide Biotechnology Intitiative from 2002-2004, where we initiated a Sloan Foundation-funded Professional Master's program. From 2005, I took on the Co-Directorship of the Nanomedicine Education and Research Consortium (NERC), which was started after receiving funding for the National Science Foundation's Interdisciplinary Graduate Education, Research and Training (IGERT) pre-doctoral training grant in Nanomedicine Science and Technology. From 2005-2009, I served as the Associate Department Chair and then took the Chair position from 2009. All of these administrative and leadership positions have helped me in being a better professional colleague and mentor.
Which individuals most influenced your research career?

As a pharmacy student at Northeastern in the late 80s, I had excellent teachers and was able to participate in undergraduate research experiences. Once I went to Purdue for $\mathrm{PhD}$ studies, I had the distinct privilege of working in Professor Kinam Park's group. Both Kinam and his wife Haesun invest a lot in their students. Their passion for science, ability to find innovative solutions, and a genuine interest in their students' successes have been essential elements in my success. Kinam also has a terrific sense of humor that makes even the most difficult day in a graduate student's life easier. After I left Purdue in 1992, I worked under the late Professor Joseph Robinson at Columbia Research Labs in Madison, WI for a short period of time. Joe was also instrumental in shaping my scientific career.

Working with Professor Bob Langer and Dr. David M. Lynn during my sabbatical appointment at MIT was one of the most remarkable experiences of my life. Bob is a world leader in biomaterials and drug delivery area, and David pioneered the concept of combinatorial design and synthesis of polymeric materials that can be screened in high-throughput fashion. In 1998, Professor Vladimir Torchilin came to Northeastern from Mass General Hospital. His appointment as Chair of Pharmaceutical Sciences Department was instrumental in our growth and visibility. Vladimir and I have had several collaborations over the years, and he has been a very effective mentor for both my scientific and professional development.

Above all, the postdocs and graduate students who have come to my group have also had a very important contribution in my personal and professional development.

Pharmaceutical scientists are faced with the dilemma of having to publish in biomedical or basic science journals. Does this mean cutting-edge science will not likely be featured in the pharmaceutical research?

Not necessarily. I believe Pharmaceutical Research provides a unique publishing platform not only to applied pharmaceutical scientists, but also to basic scientists, who can support their studies with a more translational focus, and clinical researchers interested in a diverse audience. The leadership of Pharmaceutical Research has to remain vigilant towards new areas of research and continue the traditions of the past in promoting the journal to a broad audience, while still striving to publish the highest quality manuscripts.

Where is the field of nanomedicine for cancer therapy going, and how do the articles in this theme section fill the gap?

With support from the NCI Alliance for Nanotechnology in Cancer and other public and private sources, nanotechnology 
applications for cancer diagnosis, imaging, and therapy have taken a huge leap forward over the last few years. Iron oxide nanoparticle-based magnetic resonance imaging agents (e.g. Feridex $\left.{ }^{\circledR}\right)$ are routinely used in the clinic. From the therapeutic side, Doxil ${ }^{\circledR}$ (doxorubicin in long circulating liposomes) and Abraxane ${ }^{\circledR}$ (paclitaxel in albumin nanoparticles) are some of the early trailblazers that have been approved primarily because the toxicity of the parent drugs was reduced with these nano-delivery systems.

The future of Nanomedicine for Cancer Therapy looks very bright. There have been significant recent breakthroughs in cancer biology, but these have not necessary led to development of cancer-specific therapeutics. Nanotechnology can support current cytotoxic therapies by enhancing their tumor-targeting potential and reducing systemic toxicity. Many of the cancer therapeutics also require intracellular and subcellular localization for effectiveness. Passive- and active-targeted nanoparticles can facilitate drug delivery and residence at the tumor mass. Enhancing residence is especially critical in reducing drug resistance. One can also envision several types of combination therapies encapsulated in nanoparticles with the possibility of temporal control over the release profiles. A number of important challenges such as overcoming drug toxicity and delivery of siRNA and other molecular therapies can be realized. Lastly, the use of multifunctional nanosystems with both imaging and therapeutic components can promote personalized cancer therapy through observation of drug delivery efficiency and therapeutic response for individual patients in real time.

What are the challenges for nanomedicine for cancer therapy, and how can they be overcome?

Approval of cancer nanomedicines in the U.S. and elsewhere requires thorough evaluation of safety and efficacy. Investigators working in the field should pay special attention to the safety aspect of the nanotechnology used in development of cancer nanomedicines. Biodegradable and biocompatible nanosystems should be chosen before non-degradable inorganic or carbon-based structures. Preferential accumulation in any organ system in the body upon systemic administration should raise red flags and potential toxicity concerns. Scale and manufacturing under Current Good Manufacturing Practices guidelines is necessary for any concept to move beyond the confines of preclinical evaluations to the clinic. Complicated nanosystems, especially with multiple functionalities, will be difficult to scale up and achieve the rigorous quality control necessary for drug approval. Lastly, the cost of nanotechnology-based drug development may be higher based on the need to evaluate acute and chronic safety profiles of the vehicle and even some components in both preclinical and clinical settings.

One possible solution to the challenges is to develop strong collaborations with clinicians and cancer researchers from clinical oncology programs early in the development of nanomedicines for cancer therapy. Realization of the challenges in adopting new therapeutic paradigms in the clinic is very important to scientists and engineers working in the nanotechnology field. Another area of note is continued support of NGI to insure that research in this area is encouraged. Additionally, the Alliance for Nanotechnology in Cancer can support further development by fostering additional public-private partnerships and programs like the NCL.

\section{What is the key to developing successful collaborative relationships?}

Successful research collaboration is a two-way street. It requires partnerships that are beneficial to all parties and the ability to listen to one another and act in the best interest of all concerned. In my case, the best collaborations have been with investigators who share a common interest and are dedicated to the project. The role of each collaborator should be well defined, and the expectations should be laid out in advance. Although on many occasions financial incentives are also used as a "carrot" to encourage collaborations, I have noted that these are not sustainable, especially once the funding runs out.

\section{What is your philosophy of educating graduate students?}

My approach to training pharmaceutical sciences graduate students focuses on mastery of the fundamentals of physicochemical and biological sciences with strong emphasis on problem solving through an interdisciplinary collaborative approach. The team approach to scientific problem solving should be further expanded in academic settings, and the traditional "silos" need to come down. Students should also have an opportunity to interact with their peers in other scientific disciplines and exchange ideas. Engaging with pharmaceutical/biotech industry through collaborative exchange of students for short-term research internships can also add tremendously to the success of graduate students. After graduating, students should continue this philosophy and remain malleable throughout their careers. This continuous adaptation or plasticity is the key to success in demanding research careers of the twenty-first century.

\section{What are the challenges facing the pharmaceutical sciences?}

Continuous financial support through federal sources such as NIH is critical to the success of pharmaceutical scientists, 
especially for junior faculty members who are just starting their academic careers. In the past, some of the pharmaceutical sciences disciplines, such as drug delivery, did not receive adequate federal financial support. Recently, there is more emphasis on translational biomedical science, so there is support in drug delivery. The current financial climate in pharmaceutical and biotechnology industry affects hiring of postdocs and graduate students. The frozen drug pipeline and the consequence of restructuring through major acquisitions have had a negative impact on placement of newly trained scientists.

\section{What is the place for collaboration with industry in academia?}

The current industrial climate offers tremendous opportunities for academic collaborations. Many large pharmaceutical companies are touting the new "open source" research model. I have not seen any evidence of implementation of this model, but the message has been very positive that it will lead to greater collaborative opportunities.

Partnerships with smaller companies are easier to foster than with large pharmaceutical companies due to the ease in navigating through the bureaucracy. Smaller companies may be interested in sponsored research agreements, licensing patented technologies, or short-term use of equipment and resources.

Academic scientists should also foster greater partnerships with industry through various education models that encourage industrial scientists to participate early in the training of graduate students. One example we have adopted at Northeastern is to encourage a "non-traditional $\mathrm{PhD}$ " option for students working in industry who have an MS degree. These students can do their dissertation research under a co-mentorship relationship between the faculty member and the supervisor in industry, while still keeping their day jobs. Many of the MS courses are transferred, and the student's time to graduate is decreased to about three years.

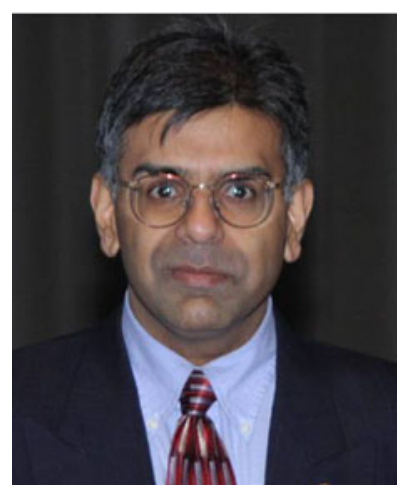

Mansoor M. Amiji, RPh,

Ph.D., received his undergraduate degree in pharmacy from Northeastern University in 1988 and his Ph.D. in pharmaceutics from Purdue University in 1992. His areas of specialization include polymeric biomaterials, advanced drug delivery systems, and nanomedical technologies.

Dr. Amiji's research interests include synthesis of novel polymeric materials for medical and pharmaceutical applications; surface modification of cationic polymers by the complexation-interpenetration method to develop biocompatible materials; preparation and characterization of polymeric membranes and microcapsules with controlled permeability properties for medical and pharmaceutical applications; target-specific drug and vaccine delivery systems for gastrointestinal tract infections; localized delivery of cytotoxic and anti-angiogenic drugs for solid tumors in novel biodegradable polymeric nanoparticles; intracellular delivery systems for drugs and genes using target-specific, long-circulating, biodegradable polymeric nanoparticles; gold and iron-gold core-shell nanoparticles for biosensing, imaging, and delivery applications. His research has received sustained funding from the National Institutes of Health (NIH), National Science Foundation (NSF), other foundations, and local industries.

Dr. Amiji is currently Distinguished Professor and Chairman of the Pharmaceutical Sciences Department and Co-director of Northeastern University Nanomedicine Education and Research Consortium (NERC). NERG oversees a doctoral training grant in Nanomedicine Science and Technology that was co-funded by the NIH and NSF. He has three published books: Applied Physical Pharmacy (McGraw-Hill, 2003), Polymeric Gene Delivery: Principles and Applications (CRC Press, 2005), and Nanotechnology for Cancer Therapy (CRG Press, 2007), along with over 150 book chapters, peer-reviewed manuscripts, and abstract publications. Dr. Amiji has received a number of awards, including the 2006 NSTI Award for Outstanding Contributions towards the Advancement of Nanotechnology, Microtechnology, and Biotechnology, and the 2007 American Association of Pharmaceutical Scientist's Meritorious Manuscript Award.

Dr. Amiji has supervised research efforts of over 80 post-doctoral associates, doctoral and master's level graduate students, and undergraduate honors students over the last 17 years. His teaching responsibilities are in Doctor of Pharmacy (Pharm.D.) program and graduate programs (M.S. and Ph.D.) in Pharmaceutical Sciences, Biotechnology, and Nanomedicine. 